\title{
Clinical Characteristics and Antimicrobial Susceptibility of Elderly Women with Acute Pyelonephritis
}

\author{
Ji Kang Yun, Woong Bin Kim, Sang Wook Lee, Kwang Woo Lee, Jun Mo Kim, Young Ho Kim
}

Department of Urology, Soonchunhyang University Bucheon Hospital, Bucheon, Korea

Purpose: Acute pyelonephritis in older patients can lead to a poorer treatment response and prognosis. The objective of this study was to classify older female patients with acute pyelonephritis according to age and compare their clinical characteristics and antibiotic susceptibility.

Materials and Methods: In this retrospective study, 360 patients aged 65 years or older and hospitalized for acute pyelonephritis in a single tertiary medical facility from 2012 to 2016 were selected as research subjects. The patients were divided into three groups according to their age. Their clinical symptoms, blood tests and urinalysis, underlying diseases, causative organisms for urinary tract infections (UTIs), and antibiotic susceptibility were compared.

Results: One hundred and seventy-six patients aged 69.9 \pm 2.7 years, 142 patients aged 79.4 \pm 3.1 years, and 42 patients aged $87.3 \pm 3.1$ years were assigned into the young-old, old-old, and oldest-old groups, respectively. The duration of hospitalization and fever due to acute pyelonephritis increased with age. On the other hand, the maximum body temperature was similar among the three groups. In blood tests, erythrocyte sedimentation rate and C-reactive protein increased with age. Among the underlying diseases, the incidence of diabetes and chronic renal disease was similar among the three groups, while cerebrovascular diseases, heart failure, and urinary stones were associated with aging. Older patients were more likely to have UTIs over the past year. Older groups showed a more significant decrease in sensitivity to carbapenems, amikacin, and ciprofloxacin.

Conclusions: Choosing appropriate antibiotics, considering the patient's age, is important when treating acute pyelonephritis.

Keywords: Pyelonephritis; Aged; Uropathogen; Susceptibility

Copyright $\subset$ 2020, Korean Association of Urogenital Tract Infection and Inflammation. All rights reserved. (c) (1) (\$) This is an open access article distributed under the terms of the Creative Commons Attribution (C) (1) (5) Non-Commercial License (http://creativecommons.org/licenses/by-nc/4.0) which permits unrestricted non-commercial use, distribution, and reproduction in any medium, provided the original work is properly cited.
Received: 8 August, 2020

Revised: 15 October, 2020

Accepted: 30 November, 2020

\author{
Correspondence to: Woong Bin Kim \\ (iD https://orcid.org/0000-0002-7369-490X \\ Department of Urology, Soonchunhyang University \\ Bucheon Hospital, 170 Jomaru-ro, Wonmi-gu, \\ Bucheon 14584, Korea \\ Tel: +82-32-621-6827, Fax: +82-32-621-5018 \\ E-mail: woongbins@schmc.ac.kr
}

\section{INTRODUCTION}

Acute pyelonephritis (APN) is a highly prevalent disease that is most likely to require hospitalization due to various symptoms, including fever [1]. Approximately half of all women will experience at least one episode of urinary tract infections (UTIs) during their lifetime, including APN.
Moreover, one in four of them experience recurrent infections [2]. In the United States, approximately 250,000 patients visit offices, and 200,000 are hospitalized each year [3]. The annual incidence is $15-17$ per 10,000 people, with a higher incidence in women than men ( 80\%) [4]. In South Korea, the annual incidence is $35.7-39.1$ cases per 10,000 people $[5,6]$. 
The symptoms of APN are diverse, including mild abdominal pain, urinary symptoms, fever, and life-threatening sepsis. A poor prognosis for patients with APN is associated with old age, immunosuppression, healthcare-related infections, obstructive uropathy, reduced platelet counts and serum albumin levels, elevated levels of C-reactive protein (CRP), and bacteremia [7-10]. Among these prognostic factors, particularly old age, the population is aging rapidly worldwide. In 2004, the population of those aged 65 or more was 461 million, which is estimated to increase to 1.5 billion by 2050 . The Republic of Korea also showed a sharp increase in the population aged 65 or over (from $7.2 \%$ in 2000 to $11 \%$ in 2010). This growth rate will continue to rise to $21.4 \%$ by 2026 and $46.5 \%$ by 2067 . Because of this tendency, age is a very important factor when treating APN patients.

APN is treated with the appropriate antibiotics for the causative organisms. In Korea, reported antibiotic resistance rates of trimethoprim/sulfamethoxazole (SXT), fluoroquinolones (FQs), third-generation cephalosporins (3rd CEPs), and carbapenems (imipenem and meropenem) were $27.8 \%, 21.8 \%, 9.3 \%$, and $0.4 \%$, respectively, during 2010-2012 [11]. Therefore, it is important to consider the patient's age, underlying disease, and clinical characteristics to select the appropriate antibiotics and prevent antibiotic resistance. This study examined the clinical characteristics of APN in older patients, as well as the differences in clinical features, causative organisms for UTIs, and susceptibility to antibiotics according to age.

\section{MATERIALS AND METHODS}

In this retrospective study, 360 patients aged 65 years or older who were hospitalized for APN in a single tertiary medical facility from 2012 to 2016 were selected as research subjects. The patients' data were recorded in a retrospective database that had been approved by Soonchunhyang University Bucheon Hospital Institutional Review Board (approval no. SCHBC 2020-05-035). Informed consent was waived because of the study design. APN was diagnosed when it met the following criteria: 1) the body temperature of a patient was above $38^{\circ} \mathrm{C}$, or the patient had a chill; 2) the patient complained of flank pain or tenderness in the costovertebral angle on a physical examination; and 3) the result of urine culture test was positive and the patient had urinary symptom (two or more of the following features: dysuria, frequent urination, urgency, and suprapubic pain). Elderly patients aged 65 years or over were classified into three age groups according to the classification criteria of the World Health Organization (WHO): young-old (between 65 and 74), old-old (between 75 and 84), and oldest-old (85 or over). The duration of hospital stay, duration of fever, and maximum body temperature of the patients were investigated based on the clinical records. The results of the laboratory tests of blood and urine were also analyzed. The data of comorbidities, prior history of UTIs, and history of previous urological intervention were obtained from their medical records and compared by age.

To collect urine for the urine culture test, the area around the external urethral orifice was cleaned using boric acid and normal saline. The midstream urine was collected in a sterile plastic cup with a lid. A $0.001 \mathrm{ml}$ sample of urine was inoculated into a blood agar and MacConkey agar plate. Each inoculated plate was incubated at $37^{\circ} \mathrm{C}$ for $18-24$ hours, and the number of bacteria grown on each medium per $\mathrm{ml}$ of urine was calculated. When more than 105 colonies were cultured per $\mathrm{ml}$ of isolated and bacteria were identified, they were regarded as the causative organisms of the UTI.

The categorical variables were compared using a Pearson chi-square test, while the continuous variables were assessed with one-way analysis of variance. IBM SPSS Statistics version 20.0 (IBM Co., Armonk, NY, USA) was used for all statistical analysis. A p-value $<0.05$ was considered significant.

\section{RESULTS}

\section{Clinical Features}

Of the 360 patients, 176 patients aged 65 to 74 (mean age: $69.9 \pm 2.7$ years), 142 patients aged 75 to 84 (mean age: $79.4 \pm 3.1$ years), and 42 patients aged 85 or over (mean age: $87.3 \pm 3.1$ years) were assigned to the young-old, old-old, and oldest-old groups, respectively. The period of hospitalization due to APN was $7.0 \pm 1.7$ days in the young-old group, $7.8 \pm 2.6$ days in the old-old group, and 12.6 \pm 2.9 days in the oldest-old group. The maximum body temperature was similar in the three groups. In the blood tests and urinalysis, the erythrocyte sedimentation rate (ESR) and CRP level differed significantly by age. On the other hand, there was no significant difference in the leukocyte count or hematuria status among the groups. 
Table 1. Clinical characteristics and laboratory findings of elderly women with acute pyelonephritis

\begin{tabular}{|c|c|c|c|c|}
\hline Characteristic & Young-old (65-74) & Old-old (75-84) & Oldest-old (>85) & $\mathrm{p}$-value \\
\hline Number & 176 & 142 & 42 & \\
\hline Age $(y)$ & $69.9 \pm 2.7$ & $79.4 \pm 3.1$ & $87.3 \pm 3.1$ & $<0.05^{\mathrm{a})}$ \\
\hline \multicolumn{5}{|l|}{ Clinical progress } \\
\hline Days of hospitalization & $7.0 \pm 1.7$ & $7.8 \pm 2.6$ & $12.6 \pm 2.9$ & $<0.05^{\mathrm{a})}$ \\
\hline Duration of fever & $3.2 \pm 1.0$ & $3.8 \pm 0.8$ & $5.1 \pm 1.6$ & $<0.05^{\mathrm{a})}$ \\
\hline Peak fever $\left({ }^{\circ} \mathrm{C}\right)$ & $39.0 \pm 1.2$ & $39.3 \pm 0.9$ & $39.0 \pm 0.6$ & $0.061^{\mathrm{a})}$ \\
\hline \multicolumn{5}{|l|}{ Laboratory findings } \\
\hline White blood cell & $16,811.4 \pm 4,888.4$ & $17,833.5 \pm 5,516.8$ & $17,775.5 \pm 6,066.0$ & $0.196^{\mathrm{a})}$ \\
\hline ESR & $97.1 \pm 20.3$ & $107.0 \pm 25.2$ & $109.7 \pm 30.5$ & $<0.05^{\mathrm{a})}$ \\
\hline C-reactive protein & $11.6 \pm 4.7$ & $13.3 \pm 5.0$ & $15.7 \pm 5.2$ & $<0.05^{\mathrm{a})}$ \\
\hline Urine $\mathrm{RBC}$ & $119(67.6)$ & $92(64.8)$ & $26(61.9)$ & $0.739^{\text {b) }}$ \\
\hline \multicolumn{5}{|l|}{ Comorbidity } \\
\hline Diabetes mellitus & $48(27.3)$ & $51(35.9)$ & $14(33.3)$ & $0.245^{b)}$ \\
\hline Cerebrovascular disorder & $25(14.2)$ & $35(24.6)$ & $12(28.6)$ & $<0.05^{\mathrm{b})}$ \\
\hline Congestive heart failure & $15(8.5)$ & $25(17.6)$ & $14(33.3)$ & $<\left.0.05\right|^{\text {b) }}$ \\
\hline Chronic kidney disease & $13(7.4)$ & $21(14.8)$ & $3(7.1)$ & $0.075^{\mathrm{b})}$ \\
\hline Chronic liver disease & $10(5.7)$ & $17(12.0)$ & $4(9.5)$ & $0.135^{\mathrm{b})}$ \\
\hline Chronic lung disease & $25(14.2)$ & $32(22.5)$ & 5 (11.9) & $0.092^{b)}$ \\
\hline Malignancy & $19(10.8)$ & $12(8.5)$ & $2(4.8)$ & $0.443^{b)}$ \\
\hline Neurogenic bladder & $32(18.2)$ & $19(13.4)$ & $5(11.9)$ & $0.394^{\text {b) }}$ \\
\hline Stone disease & $25(14.2)$ & $34(23.9)$ & $13(31.0)$ & $<0.05^{\mathrm{b})}$ \\
\hline Catheter indwelling & $17(9.7)$ & $17(12.0)$ & $6(14.3)$ & $0.634^{\text {b) }}$ \\
\hline \multicolumn{5}{|l|}{ Past history (within one year) } \\
\hline Recent urinary tract infection & $10(5.7)$ & $23(16.2)$ & $14(33.3)$ & $<0.05^{\mathrm{b})}$ \\
\hline Recent urologic manipulation & $10(5.7)$ & $8(5.6)$ & $2(4.8)$ & $0.972^{\mathrm{b})}$ \\
\hline Presence of cUTI factors & $98(55.7)$ & $86(60.6)$ & $25(59.5)$ & $0.667^{b)}$ \\
\hline
\end{tabular}

Values are presented as number only, mean \pm standard deviation or number (\%).

ESR: erythrocyte sedimentation rate, RBC: red blood cell, cUTI: complicated urinary tract infection.

${ }^{a}$ Analysis of variance. ${ }^{\text {b }}$ Pearson chi-square test.

Among the underlying diseases, cerebrovascular disease, heart failure, and urinary stones were significantly higher in the older age group. On the other hand, the incidences of diabetes, renal failure, chronic liver disease, chronic lung disease, malignant tumor, neurogenic bladder, and catheter use were similar in the three groups. For UTIs over the past year, there were 10 cases $(5.7 \%)$ in the young-old group, 23 cases $(16.2 \%)$ in the old-old group, and 14 cases (33.3\%) in the oldest-old group. The history of urologic intervention or surgery over the past year was similar in the three groups (Table 1).

\section{Causative Organisms}

Gram-negative bacteria were cultured in 327 (90.8\%) cases, while Gram-positive bacteria were cultured in 33 (9.2\%) cases. Among the Gram-negative bacteria, Escherichia coli was the most commonly detected causative organism, which was observed in 213 (59.2\%) of the 360 cases. Forty-five (12.5\%) cases of Klebsiella, 17 (4.7\%) cases of Proteus, 15 (4.2\%) cases of Pseudomonas, 15 (4.2\%) cases of Enterobacter, 12 (3.3\%) cases of Serratia, and 10 (2.8\%) cases of Citrobacter were encountered. Among the Gram-positive bacteria, there were 13 (3.6\%) cases of Staphylococcus, $11(3.1 \%)$ cases of Streptococcus, and 9 (2.5\%) cases of Enterococcus. E. coli was found in 64.2\%, $57.8 \%$, and $42.9 \%$ of the young-old group, old-old group, and oldest-old group, respectively, showing a lower incidence with increasing age. On the other hand, the cultured incidence of Proteus (4.6\% vs. 3.5\% vs. 9.5\%), Pseudomonas (3.4\% vs. $4.2 \%$ vs. $7.1 \%$ ), and Enterobacter (2.8\% vs. $4.2 \%$ vs. $9.5 \%)$ were significantly higher in the older groups (Table 2).

\section{Antibiotic Susceptibility}

Among the strains obtained from urine culture, the antibiotic susceptibility test using Gram-negative strains revealed $97.2 \%$ and $97.6 \%$ of them to show high susceptibility to ertapenem and imipenem (carbapenem antibiotics), respectively; $71.9 \%, 67.3 \%$, and $64.8 \%$ of them showed relatively high susceptibility to amikacin, ciprofloxacin, and 
Table 2. Distribution of uropathogens by age

\begin{tabular}{lcccc}
\hline \multicolumn{1}{c}{ Organism } & Total & Young-old $(65-74)$ & Old-old $(75-84)$ & Oldest-old $(>85)$ \\
\hline Gram-negative & & & & \\
Escherichia coli & $213(59.2)$ & $113(64.2)$ & $82(57.8)$ & $18(42.9)$ \\
Klebsiella & $45(12.5)$ & $23(13.1)$ & $17(12.0)$ & $5(11.9)$ \\
Proteus & $17(4.7)$ & $8(4.6)$ & $5(3.5)$ & $4(9.5)$ \\
Pseudomonas & $15(4.2)$ & $6(3.4)$ & $6(4.2)$ & $3(7.1)$ \\
Enterobacter & $15(4.2)$ & $5(2.8)$ & $6(4.2)$ & $4(9.5)$ \\
Serratia & $12(3.3)$ & $5(2.8)$ & $4(4.2)$ & $1(2.4)$ \\
Citrobacter & $10(2.8)$ & $4(2.3)$ & $6(4.2)$ & $2(4.8)$ \\
Gram-positive & & $5(2.8)$ & $5(3.5)$ & $2(4.8)$ \\
Staphylococcus & $13(3.6)$ & $4(2.3)$ & $5(3.5)$ & $2(4.8)$ \\
Streptococcus & $11(3.1)$ & $3(1.7)$ & & $1(2.4)$ \\
Enterococcus & $9(2.5)$ & & & \\
\hline
\end{tabular}

Values are presented as number (\%).

Table 3. Antimicrobial susceptibility of uropathogens from elderly women with acute pyelonephritis

\begin{tabular}{|c|c|c|c|c|}
\hline \multirow{2}{*}{ Antimicrobial } & \multicolumn{3}{|c|}{ Susceptibility } & \multirow{2}{*}{$\mathrm{p}$-value } \\
\hline & Young-old (65-74) & Old-old (75-84) & Oldest-old (>85) & \\
\hline Ampicillin & $57 / 174(32.8)$ & 40/138 (29.0) & $11 / 42(26.2)$ & 0.626 \\
\hline Cefepime & 119/175 (68.0) & $88 / 136(64.7)$ & 23/36 (63.9) & 0.789 \\
\hline Cefotaxime & $123 / 176(69.9)$ & $92 / 142(64.8)$ & 29/42 (69.0) & 0.615 \\
\hline Ceftazidime & $127 / 176(72.2)$ & $102 / 140(72.9)$ & $28 / 40(70.0)$ & 0.939 \\
\hline Gentamicin & 119/168 (70.8) & 107/142 (75.4) & $31 / 42(73.8)$ & 0.666 \\
\hline Cefazolin & $119 / 174(68.4)$ & $79 / 141(56.0)$ & $21 / 39(53.8)$ & $<0.05$ \\
\hline Ertapenem & $169 / 170(99.4)$ & $135 / 142(95.1)$ & $37 / 39(94.9)$ & $<0.05$ \\
\hline Piperacillin/tazobactam & $156 / 173(90.2)$ & $119 / 139(85.6)$ & $30 / 37(81.1)$ & 0.229 \\
\hline Amoxicillin/clavulanic acid & $111 / 176(63.1)$ & 86/135 (63.7) & 24/39 (61.5) & 0.969 \\
\hline Amikacin & $167 / 172(97.1)$ & $131 / 143(91.6)$ & $33 / 39(84.6)$ & $<0.05$ \\
\hline Cefoxitin & $153 / 176(86.9)$ & $106 / 142(74.6)$ & $28 / 40(70.0)$ & $<0.05$ \\
\hline Ciprofloxacin & $120 / 176(68.2)$ & $82 / 141(58.2)$ & $20 / 42(47.6)$ & $<0.05$ \\
\hline Imipenem & $172 / 176(97.7)$ & $131 / 142(92.3)$ & 38/42 (90.5) & $<0.05$ \\
\hline Trimethoprim/sulfamethoxazole & $107 / 176(60.8)$ & $89 / 141(63.1)$ & $26 / 42(61.9)$ & 0.914 \\
\hline
\end{tabular}

Values are presented as number (\%).

gentamicin, respectively. Although $60.9 \%$ and $67.6 \%$ of them showed susceptibility to the 3rd CEP family antibiotics, cefotaxime and ceftazidime, respectively, the susceptibilities to cefepime and cefazolin were only $48.6 \%$ and $41.6 \%$, respectively. Furthermore, 59.6\%, 50.5\%, 43.1\%, and 19.6\% of them showed susceptibility to trimethoprim/SXT, piperacillin/tazobactam, amoxicillin/clavulanic acid, and ampicillin, respectively (Table 3). Regarding the susceptibility of E. coli to antimicrobial compounds, the susceptibility to ertapenem and imipenem was the highest (98.1\%), followed by the susceptibility to amikacin at $74.6 \%$, ciprofloxacin at 70\%, gentamicin and ceftazidime at 69\%, and trimethoprim/SXT at $65.3 \%$. Less than $50 \%$ of them showed antimicrobial sensitivity to ampicillin, amoxicillin/clavulanic acid, piperacillin/tazobactam, cefepime, cefazolin, and cefoxitin. The results of antimicrobial susceptibility to other Gram-negative bacteria were similar to those for $E$. coli. On the other hand, the resistance to carbapenem was higher in Pseudomonas than that in other Gram-negative strains. The susceptibility to ertapenem and imipenem were $73.3 \%$ and $80 \%$, respectively.

The antibiotic susceptibility for $E$. coli (cultured in the largest number from patients) was assessed according to the age group. The antibiotics to which patients showed increasing resistance with increasing age included piperacillin/tazobactam ( $57.5 \%$ vs. $41.5 \%$ vs. $38.9 \%)$, imipenem (99.1\% vs. $97.6 \%$ vs. $94.4 \%)$, ciprofloxacin $(72.6 \%$ vs. $67.1 \%$ vs. $66.7 \%$ ), ertapenem (99.1\% vs. $97.6 \%$ vs. $94.4 \%)$, amikacin ( $80.5 \%$ vs. $68.3 \%$ vs. $66.7 \%$ ), and trimethoprim/SXT (79.6\% vs. $51.2 \%$ vs. $38.9 \%)$. Of these, cefoxitin and trimethoprim/SXT showed significant differences according to the age group. For other Gram-negative bacteria, the 
Table 4. Antimicrobial susceptibility according to age/uropathogens

\begin{tabular}{|c|c|c|c|c|c|c|c|c|c|c|c|c|c|c|}
\hline \multirow{2}{*}{ Organism } & \multicolumn{14}{|c|}{ Antibiotics susceptibility (\%) } \\
\hline & AMP & $\mathrm{AC}$ & PT & $\mathrm{CP}$ & $\mathrm{CT}$ & $\mathrm{CTZ}$ & $\mathrm{CZ}$ & $\mathrm{CX}$ & GM & $\mathrm{CF}$ & EP & IP & $\mathrm{AK}$ & TS \\
\hline \multicolumn{15}{|l|}{ Escherichia coli } \\
\hline Young-old & 18.6 & 53.1 & 57.5 & 46.0 & 61.9 & 75.2 & 28.3 & 58.4 & 65.5 & 72.6 & 99.1 & 99.1 & 80.5 & 79.6 \\
\hline Old-old & 22.0 & 24.4 & 41.5 & 50.0 & 64.6 & 58.5 & 45.1 & 22.0 & 73.2 & 67.1 & 97.6 & 97.6 & 68.3 & 51.2 \\
\hline Oldest-old & 22.2 & 33.3 & 38.9 & 33.3 & 55.6 & 77.8 & 44.4 & 16.7 & 72.2 & 66.7 & 94.4 & 94.4 & 66.7 & 38.9 \\
\hline Total & 20.2 & 40.4 & 49.8 & 46.5 & 62.4 & 69.0 & 36.2 & 40.8 & 69.0 & 70.0 & 98.1 & 98.1 & 74.6 & 65.3 \\
\hline \multicolumn{15}{|l|}{ Klebsiella } \\
\hline Young-old & 8.7 & 47.8 & 60.9 & 65.2 & 56.5 & 52.2 & 69.6 & 56.5 & 56.5 & 65.2 & 100 & 100 & 73.9 & 65.2 \\
\hline Old-old & 11.8 & 70.6 & 76.5 & 70.6 & 64.7 & 58.8 & 58.8 & 41.2 & 47.1 & 70.6 & 100 & 100 & 70.6 & 41.2 \\
\hline Oldest-old & 20.0 & 20.0 & 40.0 & 40.0 & 60.0 & 80.0 & 40.0 & 20.0 & 40.0 & 80.0 & 80.0 & 100 & 60.0 & 20.0 \\
\hline Total & 11.1 & 53.3 & 64.4 & 64.4 & 60.0 & 57.8 & 62.2 & 46.7 & 51.1 & 68.9 & 97.8 & 100 & 71.1 & 51.1 \\
\hline \multicolumn{15}{|l|}{ Proteus } \\
\hline Young-old & 50.0 & 12.5 & 62.5 & 37.5 & 87.5 & 87.5 & 37.5 & 62.5 & 87.5 & 75.0 & 100 & 100 & 87.5 & 75.0 \\
\hline Old-old & 40.0 & 40.0 & 40.0 & 60.0 & 80.0 & 60.0 & 20.0 & 60.0 & 80.0 & 60.0 & 100 & 100 & 60.0 & 60.0 \\
\hline Oldest-old & 50.0 & 25.0 & 25.0 & 25.0 & 50.0 & 75.0 & 75.0 & 25.0 & 75.0 & 50.0 & 100 & 100 & 50.0 & 75.0 \\
\hline Total & 47.1 & 23.5 & 47.1 & 41.2 & 76.5 & 76.5 & 41.2 & 52.9 & 82.4 & 64.7 & 100 & 100 & 70.6 & 70.6 \\
\hline \multicolumn{15}{|l|}{ Pseudomonas } \\
\hline Young-old & 0 & 66.7 & 50.0 & 66.7 & 16.7 & 66.7 & 16.7 & 16.7 & 33.3 & 33.3 & 66.7 & 83.3 & 50.0 & 16.7 \\
\hline Old-old & 0 & 33.3 & 16.7 & 33.3 & 33.3 & 83.3 & 16.7 & 33.3 & 16.7 & 50.0 & 83.3 & 83.3 & 66.7 & 16.7 \\
\hline Oldest-old & 33.3 & 33.3 & 0 & 0 & 33.3 & 33.3 & 0 & 66.7 & 33.3 & 33.3 & 66.7 & 66.7 & 33.3 & 0 \\
\hline Total & 6.7 & 46.7 & 26.7 & 40.0 & 26.7 & 66.7 & 13.3 & 33.3 & 26.7 & 40.0 & 73.3 & 80.0 & 53.3 & 13.3 \\
\hline \multicolumn{15}{|l|}{ Enterobacter } \\
\hline Young-old & 20.0 & 80.0 & 60.0 & 60.0 & 80.0 & 80.0 & 80.0 & 60.0 & 80.0 & 80.0 & 100 & 100 & 80.0 & 60.0 \\
\hline Old-old & 16.7 & 50.0 & 33.3 & 66.7 & 66.7 & 83.3 & 83.3 & 33.3 & 83.3 & 83.3 & 100 & 100 & 66.7 & 83.3 \\
\hline Oldest-old & 25.0 & 75.0 & 50.0 & 25.0 & 50.0 & 75.0 & 25.0 & 50.0 & 50.0 & 75.0 & 100 & 100 & 50.0 & 25.0 \\
\hline Total & 20.0 & 66.7 & 46.7 & 53.3 & 66.7 & 80.0 & 66.7 & 46.7 & 73.3 & 80.0 & 100 & 100 & 66.7 & 60.0 \\
\hline \multicolumn{15}{|l|}{ Citrobacter } \\
\hline Young-old & 25.0 & 75.0 & 75.0 & 25.0 & 50.0 & 50.0 & 75.0 & 50.0 & 75.0 & 50.0 & 100 & 100 & 75.0 & 75.0 \\
\hline Old-old & 0 & 25.0 & 50.0 & 75.0 & 50.0 & 75.0 & 25.0 & 50.0 & 75.0 & 75.0 & 100 & 100 & 75.0 & 50.0 \\
\hline Oldest-old & 50.0 & 50.0 & 50.0 & 50.0 & 50.0 & 50.0 & 0 & 50.0 & 50.0 & 50.0 & 100 & 100 & 100 & 50.0 \\
\hline Total & 20.0 & 50.0 & 60.0 & 50.0 & 50.0 & 60.0 & 40.0 & 50.0 & 70.0 & 60.0 & 100 & 100 & 80.0 & 60.0 \\
\hline \multicolumn{15}{|l|}{ Serratia } \\
\hline Young-old & 20.0 & 60.0 & 80.0 & 80.0 & 40.0 & 40.0 & 60.0 & 40.0 & 60.0 & 40.0 & 100 & 100 & 60.0 & 40.0 \\
\hline Old-old & 16.7 & 33.3 & 16.7 & 16.7 & 66.7 & 66.7 & 66.7 & 50.0 & 50.0 & 50.0 & 100 & 83.3 & 50.0 & 33.3 \\
\hline Oldest-old & 0 & 0 & 0 & 0 & 100 & 100 & 100 & 100 & 0 & 0 & 100 & 100 & 0 & 0 \\
\hline Total & 16.7 & 41.7 & 41.7 & 41.7 & 58.3 & 58.3 & 66.7 & 50.0 & 50.0 & 41.7 & 100 & 91.7 & 50.0 & 33.3 \\
\hline
\end{tabular}

AMP: ampicillin, AC: amoxicillin/clavulanic acid, PT: piperacillin/tazobactam, CP: cefepime, CT: cefotaxime, CTZ: ceftazidime, CZ: cefazolin, CX: cefoxitin, GM: gentamicin, CF: ciprofloxacin, EP: ertapenem, IP: imipenem, AK: amikacin, TS: trimethoprim/sulfamethoxazole.

susceptibility to antibiotics was similar in the three groups (Table 4).

\section{DISCUSSION}

UTIs are among the most frequent diseases in the urinary system and can occur regardless of gender and age. On the other hand, women have been reported to have a 4-10 times higher prevalence of UTI than men due to anatomical differences in the urinary tract. Approximately $40-50 \%$ of adult women experience UTIs once in their lifetime, and the incidence of UTIs increases with age [12].

Old age is an important risk factor that requires hospitalization in APN patients, and it is one of the common causes of elderly deaths in hospital [13]. According to the 2009 census conducted by the National Statistical Office, $10.7 \%$ of the total population of the Republic of Korea were aged 65 or over. This is expected to reach $20.8 \%$ of the total population by 2026. The Republic of Korea has already entered an aging society, and is advancing rapidly to an aging society. This means that the national healthcare expenditure is rising sharply. According to data released by the National Health Insurance Corporation in 2014, the number of patients diagnosed with pyelonephritis in the $60 \mathrm{~s}$ or older was 42,817 , accounting for $28.4 \%$ of the total 150,611 . Of 100,000 people in the relevant age group, the 
number of patients diagnosed with pyelonephritis was 592 , 583, 624, 732, 792, 992, and 1,050 in those in their 20s, 30s, 40s, 50s, 60s, 70s, and 80s or older, respectively. Hence, the incidence (per 100,000) increases with age. Therefore, it is very important to investigate the clinical features, causative organisms, and antibiotic susceptibility of APN in older patients.

Lactobacillus is a normal bacterial strain present in the female genital organ, and has a competitive relationship with pathogens. This means that conventional normal bacteria, combined with the host's receptors, can interfere with the attachment of pathogens and compete for survival with pathogens to obtain the limited nutrients provided by the environment. When a woman reaches menopause, vaginal atrophy, and symptoms of genital dryness can occur due to a decrease in estrogen. A sudden change in the genital environment can reduce the normal flora and promote UTIs. In addition to physical weakness, older female patients after menopause are presumed to have simpler cystitis and more progressed pyelonephritis.

As a result of this study, in older patients diagnosed and hospitalized with APN, the hospitalization period was $7.0 \pm 1.7$ days, $7.8 \pm 2.6$ days, and $12.6 \pm 2.9$ days in the young-old, old-old group, and oldest-old group, respectively. The period of fever also increased significantly with age. In general, normalization of the body temperature is a determining factor of the recovery prospects and the time of discharge of patients with APN. Therefore, older patients have longer fever periods and longer periods of hospitalization. The longer period of fever with the older age is believed to be due to complex causes such as a lowering of the basal metabolism and immunity with increasing age, accompanying chronic diseases, complications, malnutrition, mental disorders (such as depression or sleep disorders), and secondary accidents, such as falls.

The ESR and CRP, as possible indicators for the severity of diseases, were also significantly higher in the older age group. Hence, the severity of APN increases with age. In the treatment of elderly pyelonephritis patients, the possibility of progression to severe disease is higher than in younger pyelonephritis patients. Therefore, more active follow-up, including additional urinalysis and urine culture tests, will be required after discharge.

The risk factors for APN include diabetes mellitus, urinary stone, congenital abnormality, pregnancy, and renal transplantation. According to Min [14], diabetes mellitus was the most common cause of APN (17.7\%), followed by urinary stone (6.9\%), congenital anomaly (2.2\%), and pregnancy (1.6\%). In this study, $31.4 \%$ of patients aged 65 or over had diabetes mellitus and a high accompanying morbidity rate. On the other hand, there was no increase in the accompanying morbidity rate with age. Diabetes and diabetic complications may lead to a shorter life expectancy. Therefore, the possibility of survival bias for patients aged 85 or over with less accompanying diseases than usual should be considered. The incidence of accompanying morbidity rate of cerebrovascular disease or heart failure increases with age. On the other hand, the higher incidence of accompanying morbidity of these chronic diseases is due to aging rather than APN.

The major causative organisms of UTI are Enterobacteriaceae. Among them, E. coli is the most common uropathogen. Recently, however, the incidence of $E$. coli has decreased while the incidence of other Gram-negative bacteria, such as Pseudomonas, Proteus, and Klebsiella, and Gram-positive bacteria, has increased $[15,16]$. In this study, E. coli was the most common causative organism. According to recent studies, the incidence of $E$. coli was $87.7 \%$ among causative pathogens for UTIs [17]. On the other hand, in this study that targeted older patients, the incidence of $E$. coli was $59.2 \%$. Further classification of the patients by age showed that the incidence of $E$. coli as causative pathogen decreased with age: $64.2 \%$ in the patient group aged from 65 to $74 ; 57.8 \%$ in the patient group aged from 75 to 84 ; and $42.9 \%$ in the patient group aged 85 or over. This was attributed to the increased frequency of opportunistic infections of other bacteria, such as Gram-positive bacteria because elderly patients have relatively lower immunity and have more comorbidities than the other age groups. This difference in the distribution of causative organisms by age as well as the increasing incidence of non- $E$. coli pathogens in older patients should be considered in the early selection of antibiotics to treat APN in older patients.

An examination of antibiotic susceptibility showed that the resistance to most antibiotics of older patients exceeded $20 \%$. Most causative pathogens were highly susceptible to ertapenem and imipenem in the carbapenem class. On the other hand, carbapenem should not be used as the primary antibiotics in all older pyelonephritic patients. FQ or broad-spectrum CEP should be administered as the primary 
antibiotics and adjusted according to the antibiotic susceptibility test. Because the severity of symptoms and the rate of antibiotic resistance are higher in older patients than in general patients, carbapenem should be used first in older patients who are likely to have sepsis or septic shock. In addition, resistance to FQ increased with age, but there was no significant difference. The resistance rate in the whole population was also increasing. Therefore, the use of FQ in elderly patients with risk factors should be limited.

In previous studies, the susceptibility of $E$. coli to trimethoprim/SXT was as low as $60.6 \%$, making it an unlikely choice for a primary antibiotic [17]. In this study, the susceptibility to $E$. coli was $65.3 \%$, similar to the results of previous research. The resistance of non-E. coli was approximately $50-80 \%$ in most cases. Therefore, it should not be used as an empirical antibiotic in elderly patients with APN.

The patients were divided into three groups: young-old (65-74), old-old (75-84), and oldest-old group (>85). All patients were initially administered empirical antibiotics according to the current guidelines, which were then changed as necessary according to the results of the blood culture and urine culture tests considering the antibiotic susceptibility. In short, the empirical antibiotic strategy is to use 3rd CEPs and FQs as the first-line. Nevertheless, the use of FQs should be limited because the resistance rate increases with age. In addition, carbapenems are highly susceptible, but considering antibiotic resistance, their primary use should be considered only in high-risk older patients or patients with a high possibility of sepsis or septic shock. Furthermore, regarding trimethoprim/SXT and cefoxitin, the resistance rate increased rapidly with age, so their primary usage should be avoided in patients over 85 years old.

This study had several limitations. First, the number of patients was small, and the number of patients in each group was different. In addition, this study was retrospective in nature. Because data were organized based on the medical records, some of the history and patient symptoms might have been missing. Moreover, most patients were older patients living in specific urban areas. Hence, only patients admitted to tertiary general hospitals were selected, making it difficult for this research to represent general community-acquired UTIs. Older people living in urban areas and older people living in rural areas are more likely to show differences due to higher accessibility to antibiotics of the former. In addition, patients referred to tertiary medical institutions were likely to have undergone primary care already. For that reason, it is expected that they might show exaggerated resistance rates. Therefore, a more precise investigation will be needed in the future through nationwide studies involving several institutions. Finally, because the study was proceeded by classifying patient age into groups, an evaluation of the severity of each patient was not included. All patients, from patients with simple APN to patients with obstructive APN who require urological intervention as an emergency, were included as subjects if they met the diagnostic criteria. Therefore, more studies will be needed to classify patients into more clear diagnostic criteria.

\section{CONCLUSIONS}

Even in acute disease, long-term hospitalization can result in a high medical cost for a patient. In addition, it may increase the incidence of complications by raising the risk of hospital-acquired infections [18]. Therefore, it is important to understand different features of APN in older patients compared to younger patients, anticipate risk factors for long-term hospitalization, and try to select the appropriate antibiotics.

\section{CONFLICT OF INTEREST}

No potential conflict of interest relevant to this article was reported.

\section{ACKNOWLEDGMENTS}

This study was supported by the Soonchunhyang University Research Fund.

\section{AUTHOR CONTRIBUTIONS}

J.K.Y. participated in data collection, performed the statistical analysis and wrote the manuscript. S.W.L., K.W.L., J.M.K. and Y.H.K. participated in the study design. W.B.K. participated in the study design and coordination and helped to draft the manuscript. All authors read and approved the final manuscript. 


\section{ORCID}

Ji Kang Yun, https://orcid.org/0000-0002-8655-2484

Woong Bin Kim, https://orcid.org/0000-0002-7369-490X

Sang Wook Lee, https://orcid.org/0000-0001-9660-4092

Kwang Woo Lee, https://orcid.org/0000-0002-8882-2492

Jun Mo Kim, https://orcid.org/0000-0003-4978-1867

Young Ho Kim, https://orcid.org/0000-0003-3959-0928

\section{REFERENCES}

1. Foxman B, Klemstine KL, Brown PD. Acute pyelonephritis in US hospitals in 1997: hospitalization and in-hospital mortality. Ann Epidemiol 2003;13:144-50.

2. Brown PD. Antibiotic selection for urinary tract infection: new microbiologic considerations. Curr Infect Dis Rep 1999;1: 384-8.

3. Czaja CA, Scholes D, Hooton TM, Stamm WE. Population-based epidemiologic analysis of acute pyelonephritis. Clin Infect Dis 2007; 45:273-80.

4. Hooton TM, Stamm WE. Diagnosis and treatment of uncomplicated urinary tract infection. Infect Dis Clin North Am 1997;11:551-81.

5. Ki M, Park T, Choi B, Foxman B. The epidemiology of acute pyelonephritis in South Korea, 1997-1999. Am J Epidemiol 2004; 160:985-93.

6. Kim B, Myung R, Kim J, Lee MJ, Pai H. Descriptive epidemiology of acute pyelonephritis in Korea, 2010-2014: population-based study. J Korean Med Sci 2018;33:e310.

7. Hsu CY, Fang HC, Chou KJ, Chen CL, Lee PT, Chung HM. The clinical impact of bacteremia in complicated acute pyelonephritis. Am J Med Sci 2006;332:175-80.

8. Lee $\mathrm{JH}$, Lee $\mathrm{YM}$, Cho JH. Risk factors of septic shock in bacteremic acute pyelonephritis patients admitted to an ER. J
Infect Chemother 2012;18:130-3.

9. Tambo M, Okegawa T, Shishido T, Higashihara E, Nutahara K. Predictors of septic shock in obstructive acute pyelonephritis. World J Urol 2014;32:803-11.

10. Yamamoto Y, Fujita K, Nakazawa S, Hayashi T, Tanigawa G, Imamura $\mathrm{R}$, et al. Clinical characteristics and risk factors for septic shock in patients receiving emergency drainage for acute pyelonephritis with upper urinary tract calculi. BMC Urol 2012;12:4.

11. Wie SH, Ki M, Kim J, Cho YK, Lim SK, Lee JS, et al. Clinical characteristics predicting early clinical failure after $72 \mathrm{~h}$ of antibiotic treatment in women with community-onset acute pyelonephritis: a prospective multicentre study. Clin Microbiol Infect 2014;20:O721-9.

12. Kunin CM. Urinary tract infections: detection, prevention, and management. 5th ed. Baltimore: Williams \& Wilkins; 1997.

13. Efstathiou SP, Pefanis AV, Tsioulos DI, Zacharos ID, Tsiakou AG, Mitromaras AG, et al. Acute pyelonephritis in adults: prediction of mortality and failure of treatment. Arch Intern Med 2003;163:1206-12.

14. Min HJ. Acute pyelonephritis: clinical study and consideration about inpatient therapy. Korean J Med 1998;55:232-44.

15. Nicolle LE. Epidemiology of urinary tract infections. Clin Microbiol Newsl 2002;24:135-40.

16. Stamm WE, Norrby SR. Urinary tract infections: disease panorama and challenges. J Infect Dis 2001;183 Suppl 1:S1-4.

17. Kim WB, Cho KH, Lee SW, Yang HJ, Yun JH, Lee KW, et al. Recent antimicrobial susceptibilities for uropathogenic escherichia coli in patients with community acquired urinary tract infections: a multicenter study. Urogenit Tract Infect 2017;12: 28-34.

18. Eveillard M, Pisante L, Mangeol A, Dolo E, Guet L, Huang M, et al. [Specific features of nosocomial infections in the elderly at a general hospital center. 5 surveys of annual prevalence]. Pathol Biol (Paris) 1998;46:741-9. French. 